\title{
Transfer effects of mixed and unmixed list designs in paired associate learning of children'
}

\author{
G. J. Johnson, UNIVERSITY OF TEXAS \\ R. K. Penney, UNIVERSITY OF WATERLOO
}

\begin{abstract}
Sixth grade children learned a list of paired associates and then were randomly assigned to one of three lists. An unmixed list (UL) was constructed of AB-CD items which would provide neutral transfer from the first to the second list. Another UL was constructed by repairing the $S-R$ components of the first list (AB-ABr) and thereby producing negative transfer. A mixed list (ML) contained half of the items from each of the two UL controls. The results of the comparison between the UL group and the $\mathrm{ML}$ group on the same $\mathrm{AB}-\mathrm{ABr}$ test pairs showed superior performance by the $M L$ group. No significant differences in performance were found on the $A B-C D$ test items as a function of $M L$ vs. UL designs.
\end{abstract}

\section{Problem}

Twedt \& Underwood (1959) investigated transfer effects as a function of mixed vs. unmixed lists in paired associate learning. The second list was the same for all Ss (college students), while the first list items were selected in such a manner as to provide four different transfer paradigms. In the unmixed list (UL) condition all second list items formed the s a m e transfer paradigm with first list items. In the mixed list (ML) design subgroups of second list items formed differe $n t$ transfer paradigms with first list items. The results showed no difference in the amount of transfer as a function of ML vs. UL design for each of the paradigms used.

On the other hand, two paired associate studies using college Ss have demonstrated differential effects of ML and $\mathrm{UL}$ designs in the situation where $\mathrm{S}$ learns a single list. Underwood \& Schulz (1960) employed three lists of either high, low or mixed dominance. The mixed dominance list was composed of half of the items from each of the high and low dominance lists. No differences were found when high dominance items in the UL and ML conditions were compared. However, the low dominance pairs were more easily learned in the mixed dominance list than in the unmixed dominance list.

Johnson (1964) using a single list design also found differential performance as a function of $M L$ or $U L$ conditions. Three paired associate lists of two syllable adjectives were employed. The strong unmixed association list (UAL) contained 12 pairs of high association items. The weak UAL consisted of 12 weakly associated pairs. The mixed association list (MAL) contained six items from each of the two UAL controls. In the MAL, performance was better on the weakly associated test pairs and worse on the strongly associated test pairs as compared to the same subsets of items in the two UAL controls.

Transfer studies using the ML procedure normally provide a situation where, due to first list training, subgroups of second list items differ to a greater or lesser extent in the strength of association between S-R components. Therefore, the conclusion that ML and UL designs can be used interchangeably in transfer studies would seem to be called into question by the results of Underwood and Schulz and by those of Johnson.

The present study was designed to further investigate transfer effects as a function of ML and UL designs using children as subjects.

\section{Method}

Twenty-one male and 21 female sixth grade students were administered a list of eight paired associate adjectives adopted from a list developed by Castaneda et al (1961). To minimize competition, the associative strength across separate S-R pairs within each list was low. All Ss learned the same first list which consisted of the following items: rough-less; free-healthy; important-bumpy; unable-jolly; kind-sleepy; lazy-thin; wettight; poor-close. A criterion of two perfect recitations or a maximum of 30 trials was imposed upon this first list.

Approximately $1 \mathrm{~min}$. after reaching criterion, a second list of paired associates was administered. For second list learning seven males and seven females were assigned to each of the three treatment groups; $\mathrm{AB}-\mathrm{CD}$, unmixed list (UL), $\mathrm{AB}-\mathrm{ABr} \mathrm{UL}$ and $\mathrm{AB}-\mathrm{CD}$ mixed list (ML). The $A B-C D$ was composed of eight pairs of adjectives which would provide neutral transfer from the first to the second list (afraid-love, foolishglad, nice-wrong, hot-careful, damp-white, early-dead, fair-back and shiny-much). The AB-ABr UL was developed by re-pairing the stimulus and response components of the first list items (rough-close, free-tight, important-thin, unable-sleepy, kind-bumpy, lazy-jolly, wet-healthy and poor-less) and thereby producing negative transfer. Finally, the ML was constructed by combining four randomly selected items from each of the two UL controls (hot-careful, *wet-healthy, shinymuch, *kind-bumpy, *free-tight, *rough-close, afraidlove, and foolish-glad). The astericks indicate those pairs that formed the $\mathrm{AB}-\mathrm{ABr}$ transfer paradigm while the remaining pairs formed the $A B-C D$ paradigm. The Ss were given a maximum of 10 trials (including the first exposure) on the second list or were terminated upon two successful recitations of the list. Both the first and second lists were presented in three different 


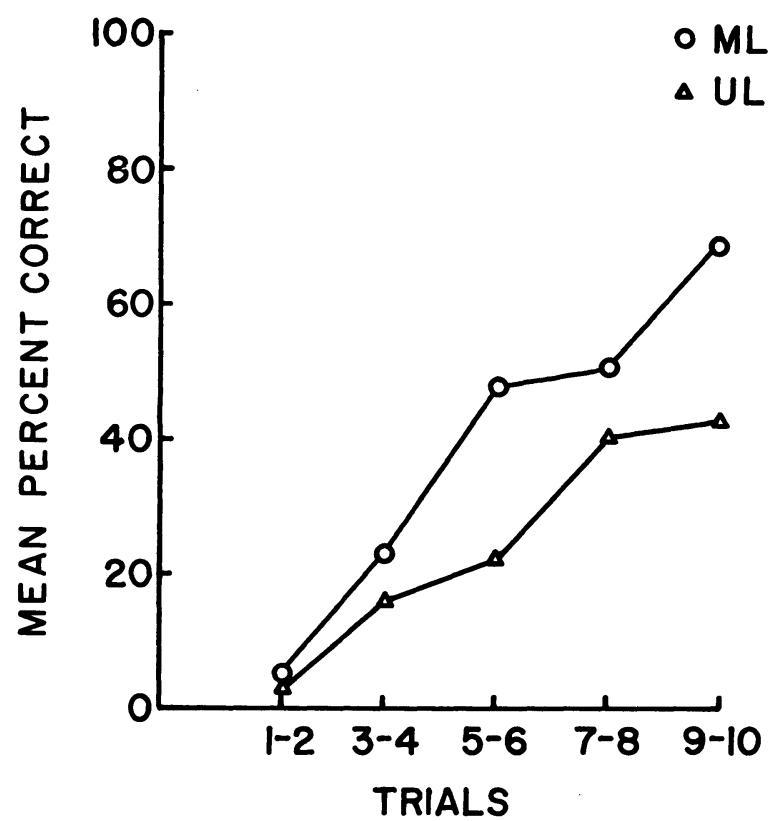

Fig. 1. Mean percent correct responses on $\mathrm{AB}-\mathrm{ABr}$ test pairs in mixed and unmixed lists.

random orders.

A Hunter Card Master exposed successive stimulus items every $4 \mathrm{sec}$. with a $4 \mathrm{sec}$. interval between successive presentations of the list. The anticipation interval was $2 \mathrm{sec}$.

\section{Results}

When an analysis of variance was applied to the total number of anticipation errors over the 30 trials of the first list, a non-significant $F$ indicated that prior to the learning of the second list, the three groups were homogeneous in ability.

The results of the comparison of performance on the AB-CD test pairs (S-R pairs common to both lists) in the UL and ML groups indicate no consistent difference between ML and UL conditions. That is, when an analysis of variance was applied to the total number of errors over five blocks of two trials each, a nonsignificant List effect was found, $F(1,24)=1.73$ for the $A B-C D$ test pairs of the ML and UL groups. However, since the ML contained only half of the items which appeared in the AB-CD UL, a further analysis was performed to determine if there was a difference in number of errors made on the $A B-C D$ test pairs and the remaining four pairs in the $A B-C D U L$. The resulting $F(<1)$ indicated that the two subsets of items were homogeneous in difficulty.

Figure 1 compares the performance curves for the $\mathrm{ML}$ group and $\mathrm{UL}$ group on the $\mathrm{AB}-\mathrm{ABr}$ test pairs common to both lists. As indicated the two groups performed at approximately the same level at trials $1-2$, but from this point on the performance of the ML group was clearly superior. An analysis of variance yielded a significant list effect, $F(1,24)=4.76 ; p<.05$, and Trials $\mathrm{x}$ List interaction, $\mathrm{F}(4,96)=3.05 ; \mathrm{p}<.05$. A further analysis failed to indicate a significant difference in total number of errors between test pairs and non-test pairs in the $\mathrm{AB}-\mathrm{ABr} \mathrm{UL}$ indicating that the two subsets of items were homogeneous in difficulty.

\section{Diseussion}

The results failed to show a difference in performance on $A B-C D$ transfer items as a function of ML vs. UL design. However, performance on $\mathrm{AB}-\mathrm{ABr}$ items was shown to be significantly better under the ML condition than under the UL condition.

The results of the present study fail to support the conclusion of Twedt and Underwood that ML and UL designs can be used interchangeably in studying transfer of training. At least two factors may be involved in this discrepancy. The first is that in the Twedt and Underwood study four different sets of items were combined in the ML while in the present study only two different ML sub-groups were provided. The second factor is a developmental variable; i.e., differences between children and adults. Keppel (1964) suggests that factors which have been isolated for adult learning may have differential influence on the verbal learning of children.

\section{References}

CASTANEDA, A., FAHEL, L. S., \& ODOM, R. Associative characteristics of sixty-three adjectives and their relation to verbal paired-associate learning in children. Child Develpm., 1961, 32, 297-304.

JOHNSON, G. J. The effects of mixed vs. unmixed list designs in paired-associate learning. Unpublished Masters thesis. University of Texas, 1964.

KEPPEL, G. Verbal learning in children. Psychol. Bull., 1964, 61, 63-80.

TWEDT, H. M., \& UNDERWOOD, B. J. Mixed vs. unmixed lists in transfer studies. J. exp. Psychol., 1959, 58, 111-116.

UNDERWOOD, B. J., \& SCHULZ, R. W. Response dominance and rate of learning paired associates. J. gen. Psychol., 1960, 62, 153-158.

Note

1. Sincere appreciation is extended to Mr. Raymond Shelton, principal of Maplewood School, Austin, Texas. 